\title{
Hypoxic preconditioning ameliorates endometrial and myometrial damage and improves uterine function following prolonged hypoxia in nonpregnant rats
}

\author{
Mohammed Alotaibi \\ Department of Physiology, College of Medicine, King Saud University, Riyadh, Kingdom of Saudi Arabia
}

\begin{abstract}
Normal repeated uterine contractions are associated with uterine hypoxic stresses, and uterine transplantation and severe bleeding during hysterectomy may lead to hypoxia and irreversible cellular damage. This study investigated the effects of short repeated hypoxic episodes on the structure and function of uterine tissues following sustained prolonged hypoxia. Small segments of uterine tissue were dissected from three groups of nonpregnant rats and mounted in a tissue bath system. Prolonged hypoxia markedly increased the infiltration of eosinophils into the myometrium and caused fibrotic stroma and degeneration of endometrial glands with marked infiltration of eosinophils into the endometrium compared to the control group. In addition, the mean myometrial contractile function significantly decreased to $69 \pm 1 \%$ compared to $100 \%$ control with irregular and uncoordinated contractile activity $(p<0.01)$. Intriguingly, preconditioning with brief hypoxic episodes prevented the endometrial and myometrial degenerative changes. Although the mean myometrial contractile function decreased to $80 \pm 3 \%$ during reoxygenation compared to the $100 \%$ control, the entire force was greater than the force in the non-preconditioned group $(p<0.01)$. These results provide compelling evidence that prolonged hypoxia exacerbates the degree of cellular damage and that preconditioning with repeated cycles of short hypoxia/reoxygenation can ameliorate cellular damage.
\end{abstract}

Key words: Contraction — Endometrium - Hypoxia - Myometrium — Nonpregnant

\section{Introduction}

The uterus is a myogenic organ composed primarily of smooth muscle cells (the myometria) which produce the actual contractions. The nature of these contractions is phasic and spontaneous which shows cycles of discrete repeated contractions and relaxation without hormonal or neural stimuli (Wray et al. 2015). Uterine contractions are necessary during transport of sperm from the cervix into the oviducts, during menstruation to expel the functional endometrial layer each month, throughout pregnancy, and importantly, during labor to expel the fetus and placenta. Anatomically, the uterus is supplied by the uterine artery, which traverses the myometrium and subdivides into small blood vessels that supply the deep endometrial layer.

Correspondence to: Mohammed Alotaibi, Department of Physiology, College of Medicine, King Saud University, P.O Box 2925, Riyadh 11461, Kingdom of Saudi Arabia

E-mail: mfalotaibi@ksu.edu.sa
Previous studies on women and animals have shown that normal uterine contractions are associated with a reduction in uterine blood flow (Brotanek et al. 1969; Larcombe-McDouall et al. 1999). As the uterus contracts, it occludes its own blood vessels, resulting in local hypoxia/ischemia, which occurs repetitively with subsequent contractions. The intensity and frequency of uterine contractions can be augmented by uterotonics such as oxytocin or prostaglandins. These are given routinely to some women during dysfunctional labors, which can further increase the intensity of uterine activity and hence the duration of hypoxia. We recently discovered that uterine tissue obtained from laboring women or rats responded more strongly to oxytocin than uterine tissues from nonpregnant or early pregnant women or rats, suggesting that the myometrium becomes progressively more sensitive to the uterotonics in preparation for labor (Alotaibi 2017). In fact, some women are much more sensitive than others to the effect of uterotonics, and uterine hypertonicity, tachysystole, or hyperstimulation can develop even with low doses (Kunt et al. 2010). 
Severe hypoxia/ischemia can occur in tissues during trauma, organ transplantation, surgery, or in the presence of tumor resulting in tissue dysfunction or irreversible cellular damage (McCully et al. 2004; Kupiec-Weglinski et al. 2005). However, abrupt restoration of blood flow/reoxygenation after prolonged ischemia/hypoxia has been reported to increase rather than decrease the cellular damage as it stimulates the production of reactive oxygen species (ROS) which can further accelerate the cellular damage and affect the functional recovery (Gottlieb et al. 1994; Gute et al. 1998). Ischemia/reperfusion injury is the main cause of uterine damage during uterine transplantation, hysterectomy for benign or malignant tumors, or during severe postpartum hemorrhage (Fageeh et al. 2002; Diaz-Garcia et al. 2013).

A new phenomenon known as hypoxic or ischemic preconditioning was first discovered by Murry et al. in 1986, where brief cycles of hypoxia/ischemia were found to markedly reduce the myocardial cellular injury following sustained hypoxia/ischemia (Murry et al. 1986). We found previously that repeated cycles of short hypoxia can significantly improve the uterine contractile activities in laboring women and pregnant rats (Alotaibi et al. 2015) and that pregnant uterus could tolerate the deleterious effects of hypoxia than the nonpregnant tissue (Alotaibi 2018). Moreover, it has been shown that prolonged hypoxia/ischemia can cause profound endothelial dysfunction and that hypoxic preconditioning (HPC) can significantly protect microvascular endothelial cells against the deleterious effects of prolonged hypoxia (Kharbanda et al. 2001; Wu et al. 2013). In the present study, we used an in vitro rat model to study the effects of HPC on isolated uterine smooth muscles.

We tested the hypothesis that sustained prolonged hypoxia can cause irreversible endometrial and myometrial damage and then tested if HPC could ameliorate this damage or improve the contractile function in nonpregnant rats.

\section{Materials and Methods}

\section{Animals and uterine tissue}

Adult female Virgin Wistar rats weighing 200 g, obtained from a local supplier, were used in this study. The experiments performed on laboratory rats were conducted in accordance with the advice and approval of the Institutional Animal Care Committee (IACC) of King Saud University. Rats were housed in a temperature-controlled room $(\sim 24$ $26^{\circ} \mathrm{C}$ ) with a 12-hour light/dark cycle and were fed food pellets and water ad libitum. All rats were in the diestrus stage of the estrous cycle as determined by a daily vaginal smear. On the day of the experiment, the animals were euthanized by cervical dislocation under $\mathrm{CO}_{2}$ inhalation according to the Schedule 1 procedure of the UK Scientific Act of 1986
The abdomen was opened longitudinally and the uterus was immediately removed and placed into a physiological saline solution (PSS) containing (in $\mathrm{mM}$ ): $115 \mathrm{NaCl}, 4.7 \mathrm{KCl}, 2$ $\mathrm{CaCl}_{2}, 1.2 \mathrm{MgSO}_{4}, 1.18 \mathrm{KH}_{2} \mathrm{PO}_{4}, 22 \mathrm{NaHCO}_{3}$, and 7.88 dextrose, (pH 7.40).

\section{Measurement of tension and experimental protocols}

Longitudinal uterine strips $(2 \times 10 \mathrm{~mm})$, with intact endometrium and serosa, were dissected carefully and mounted in $5 \mathrm{ml}$ organ bath chambers (Panlab, ADInstruments Ltd, Sydney, Australia). Uterine strips were stretched to $1 \mathrm{~g}$ standard resting tension and allowed to equilibrate in a buffered PSS solution with $100 \% \mathrm{O}_{2}$ at $37^{\circ} \mathrm{C}$ for at least $30 \mathrm{~min}$. To measure the degree of $\mathrm{O}_{2}$ or $\mathrm{N}_{2}$ and to deliver the appropriate amount needed, a sophisticated fibre-optic oxygen microprobe (OxyMicro, World Precision Instruments, FL, USA) was deeply inserted into the solution and the amount of delivered gas was manually adjusted from the tank. Once steady and regular phasic uterine contractions were established, uterine strips were divided into three groups: 1 ) Control group ( $n=$ 9), uterine strips received $100 \% \mathrm{O}_{2}$ without any hypoxia; 2) Prolonged hypoxia group $(n=9)$, uterine strips received sustained hypoxia for $40 \mathrm{~min}$ (by replacing the $\mathrm{O}_{2}$ with $\mathrm{N}_{2}$ ) followed by 20 -min reoxygenation (recovery) period and 3) Preconditioning group $(n=9)$, uterine strips received 3 cycles of 2 min hypoxia separated by recovery periods and were subjected to sustained hypoxia for $40 \mathrm{~min}$ followed by 20-min reoxygenation period.

\section{Histopathological examination}

After the 20-min reoxygenation period, the uterine strips were immediately removed from the bathing solution and fixed in $10 \%$ buffered formalin for 48 hours. The strips were then processed (Tissue processor Leica ASP300, Germany) and embedded (Leica EG1150 $\mathrm{H}$ embedder, Germany) in paraffin wax. Tissue sections $(\sim 5 \mu \mathrm{m}$ thick $)$ were stained with hematoxylin-eosin (H\&E) for morphological studies. Photomicrographs were captured at a magnification of $\times 200$ using a BX-60 light microscope equipped with a digital camera (Olympus Corporation, Tokyo, Japan) and examined for histological analysis by arbitrary scale to assess the severity of morphological changes. Histologic examination was performed by an experienced histopathologist who was blinded to the experimental groups.

\section{Data and statistical analyses}

Data were analyzed using Origin Software (OriginLab, Northampton, MA). The main contractile parameter calculated was the area under the curve (AUC) which measures the entire contractile activity under the area of interest. Data 
were analyzed as follows: contractile activity during the last 10 min in $100 \% \mathrm{O}_{2}$ (before application of hypoxia) was calculated and designated as a $100 \%$ control. The last $10 \mathrm{~min}$ of the recovery period (after the prolonged hypoxia) was then calculated and expressed as a percentage of this control. Data were presented as mean \pm standard error of the mean (SEM) using the appropriate $t$-test. $p$ values $<0.05$ were considered as statistically significant with $n$ representing the number of uterine samples, one from each rat. Histopathological alterations were graded as minimal, moderate, or severe (Schafer et al. 2018).

\section{Results}

\section{Normal uterine function and structure}

As shown in Fig. 1A, isolated uterine strips are able to produce frequent, regular, and phasic spontaneous contractions if placed in an optimal environment (i.e. continuous perfusion of PSS with $100 \% \mathrm{O}_{2}$ at $37^{\circ} \mathrm{C}$ ). In the histologic examination, the endometrial glands and stroma (lamina propria) appeared normal with an intact and regular arrangement of the myometrial cells (Fig. 1B).

\section{Effects of prolonged hypoxia on uterine function and structure}

Application of prolonged sustained hypoxia for $40 \mathrm{~min}$ decreased the entire contractile force significantly during hypoxia to $12 \pm 3 \%(p<0.0001)$, and during reoxygenation to $69 \pm 1 \%$ compared to the $100 \%$ control (before application of hypoxia) ( $n=9 ; p<0.01 ;$ Fig. $2 \mathrm{~A}$ ). In all uterine strips tested, the pattern of contractile activity did not return to the regu- lar and stable contractions seen before the induction of the hypoxia with incoordinate contractile pattern. The baseline of contractions was markedly dropped during the prolonged hypoxia and became elevated and irregular throughout the reoxygenation period. The histologic examination revealed severe degenerative changes in both endometrial and myometrial cells (Fig. 2B). The myometrial cells were markedly affected by the presence of concentric fibroid tissues around the cells and the endometrial glands showed surrounding severe fibrotic stroma with marked infiltration of eosinophils.

\section{Effects of repetitive short hypoxia on uterine function and structure after prolonged hypoxia}

Application of 3 cycles of 2 min hypoxia decreased the force of contraction and the tissue was able to recover successfully after each hypoxic episode. However, when prolonged sustained hypoxia was applied for $40 \mathrm{~min}$, the entire uterine force decreased significantly during hypoxia to $33 \pm 4 \%$ ( $p<$ 0.01 ), and during reoxygenation to $80 \pm 3 \%$ compared to the $100 \%$ control (before application of hypoxia) $(n=9 ; p<0.01$; Fig. 3A). Although the force decreased during the reoxygenation period in this group, it was more powerful than the force during reoxygenation in the prolonged hypoxia group. Histological examination revealed mild infiltration of the endometrial glands and stroma by eosinophils and mild to moderate degenerative changes in myometrial cells (Fig. 3B).

\section{Discussion}

We recently discovered that repeated episodes of transient hypoxia could significantly improve the contractile uterine
A

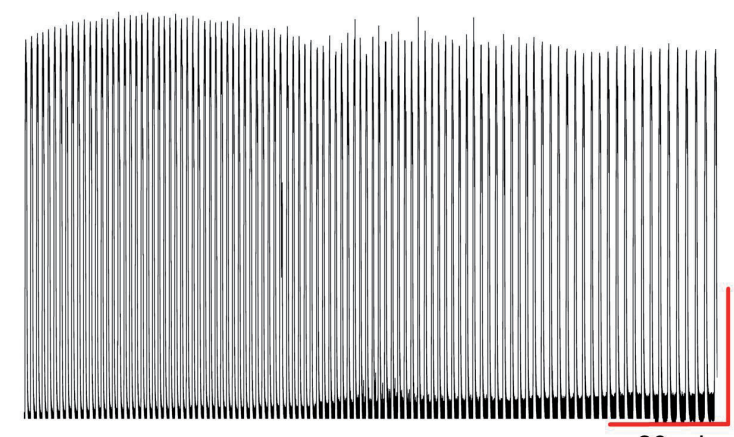

$20 \mathrm{~min}$
B

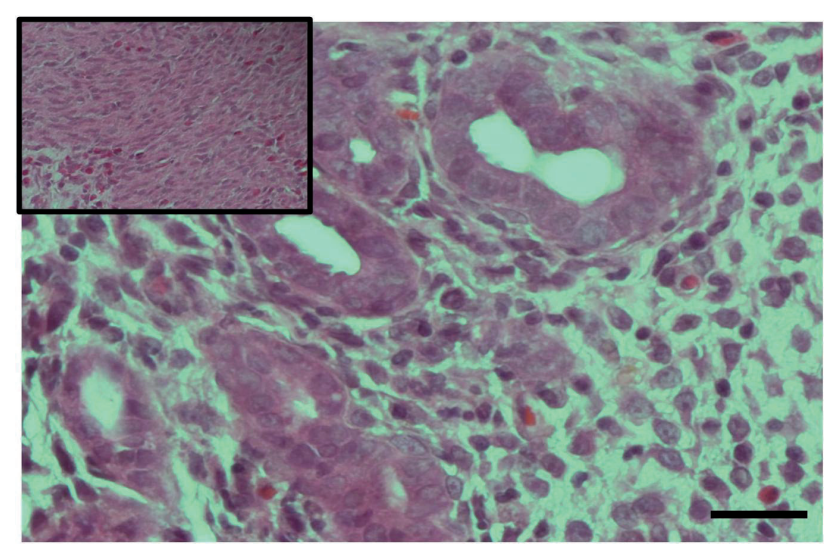

Figure 1. A. Representative original recording showing the spontaneous uterine activity in $100 \%$ oxygen. B. Normal endometrial glands and stroma (lamina propria) in rat uterus. Note the presence of normal myometrial cells (inset). Light microscopy: staining with hematoxylin and eosin $\times 200$. Scale bar represents $50 \mu \mathrm{m}$. 
A

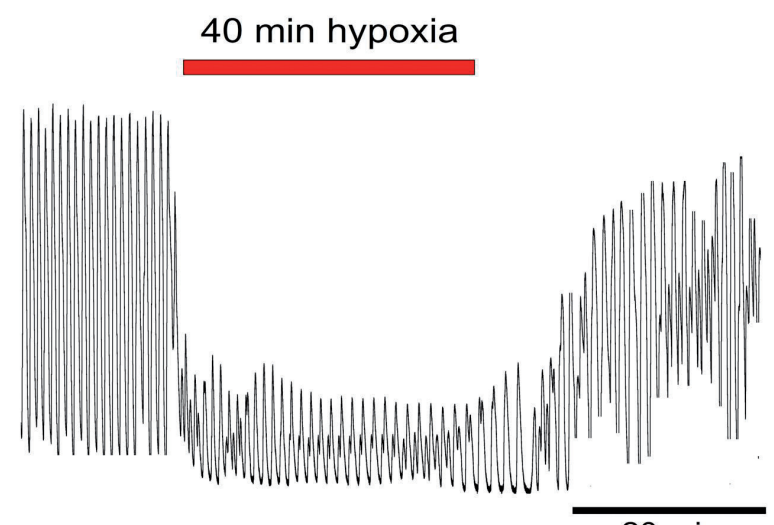

B

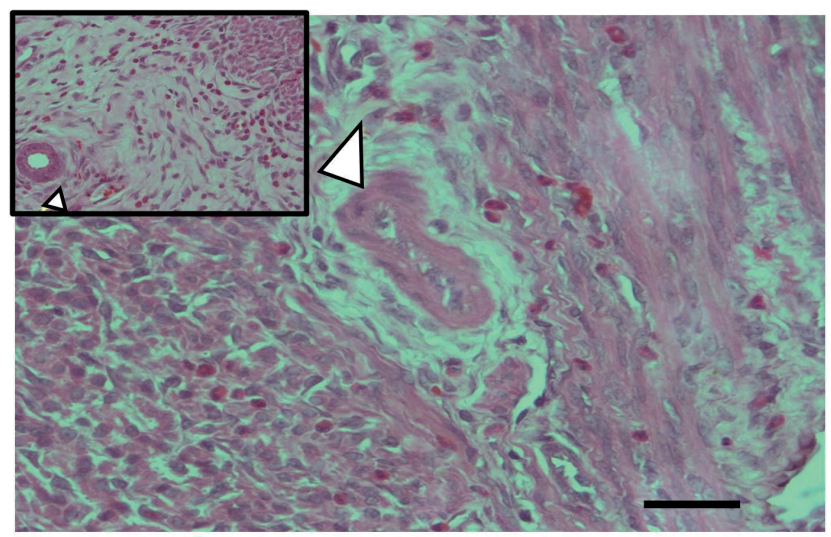

Figure 2. A. Representative original recording showing the effect of 40 min hypoxia on rat uterine contractile activity. B. Marked infiltration of numerous eosinophils into the myometrium (arrowhead). The endometrial glands appear with surrounding fibrotic stroma with marked infiltration of eosinophils (inset, arrowhead). Light microscopy: staining with hematoxylin and eosin $\times 200$. Scale bar represents $50 \mu \mathrm{m}$.

function after prolonged lethal hypoxia in the pregnant but not in the nonpregnant rats (Alotaibi 2018). The present study investigated the impact of intermittent short hypoxic episodes on the survival of endometrial and myometrial cells as well as on uterine contractile function following prolonged sustained hypoxia in nonpregnant rats. We found that sustained hypoxia can cause significant severe irreversible damage to uterine tissues, which is ameliorated by preconditioning with short, repetitive, nonlethal hypoxic episodes. The uterus is an active organ even at rest, producing cyclical phasic spontaneous contractions separated by periods of relaxation. Like any other organ in the body, it is vulnerable to some degree of pathophysiological hypoxia which is known to have a strong impact on cellular transcriptome (Cummins et al. 2005). Repeated hypoxic/ ischemic insults occur normally within the uterus as it occludes its own blood vessels during each contraction with subsequent alterations of intracellular $\mathrm{pH}$ and metabolites (Larcombe-McDouall et al. 1999). Sustained hypoxia/ischemia can also develop within the uterus during uterine transplantation, long gynecological surgeries, and during severe postpartum hemorrhage. As a result, cellular injury
A

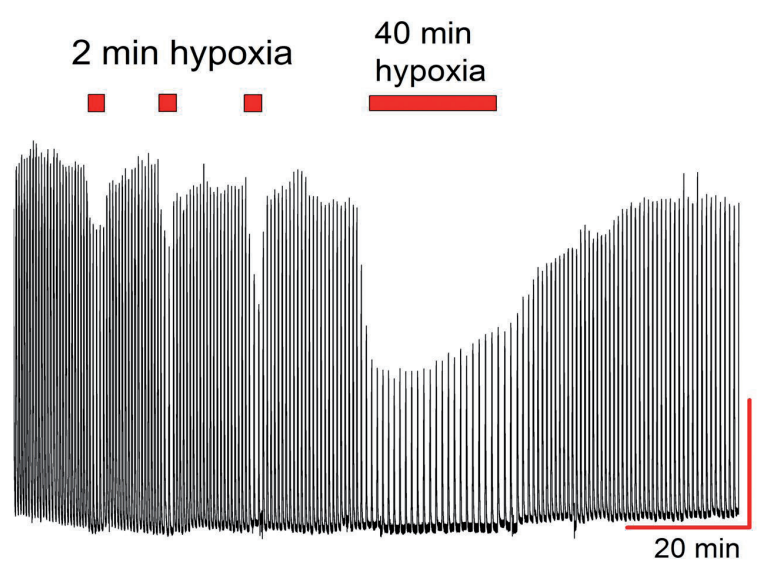

B

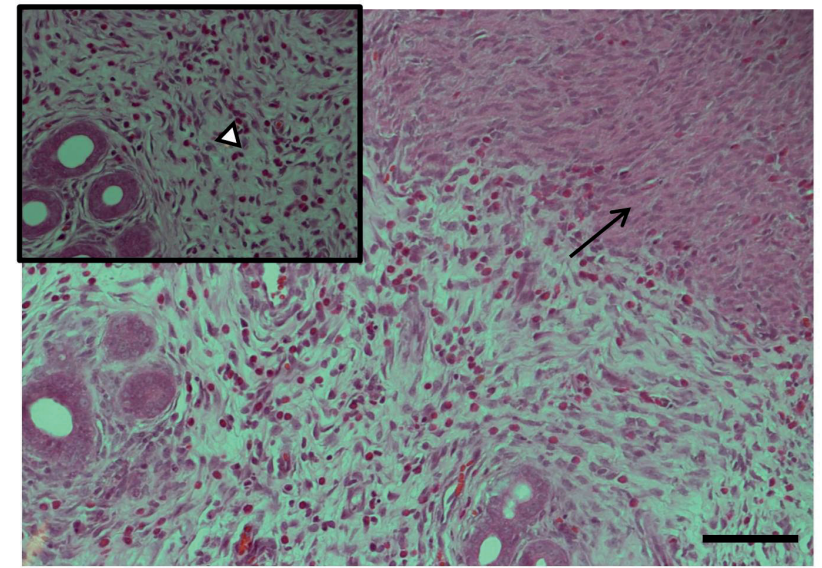

Figure 3. A. Representative isometric recording showing the effect of prolonged hypoxia (40 min) on uterine contraction preconditioned with repeated brief cycles of hypoxia in nonpregnant rats. B. Endometrial glands and stroma infiltrated by mild eosinophils (inset, arrowhead). Myometrial cells are mildly affected (arrow). Light microscopy: staining with hematoxylin and eosin $\times 200$. Scale bar represents $50 \mu \mathrm{m}$. 
or death may occur secondary to hypoxic/ischemic insults, which is correlated with the duration and intensity of blood flow interruption. During the period of sustained hypoxia/ ischemia, the stored ATP is depleted resulting in a generation of ROS during the reoxygenation/reperfusion period (Horton et al. 1993) which can directly or indirectly affect the integrity of the cellular membrane. In this study, we showed that prolonged hypoxia clearly damaged the endometrial and myometrial cells and these degenerative changes did not improve during the recovery period even after leaving the tissue in $100 \%$ oxygen for a long period, suggesting that irreversible damage to some uterine compartments took place. Our findings are consistent with previous studies in vivo which found severe degenerative endometrial changes with infiltration of leukocytes (including eosinophils) after prolonged ischemia in nonpregnant rats (Sahin et al. 2014; Aslan et al. 2017). Activated eosinophils were reported to release free radicals (Chanez et al. 1990) and were implicated in epithelial damage (Yukawa et al. 1990). The major targets of generated ROS are endothelial and smooth muscle cells which can lead to severe mitochondrial and cellular damage, and muscle dysfunction (Ballinger et al. 2000). Another typical sign of endometrial damage due to prolonged hypoxia is the destruction of endometrial glands and the presence of fibrotic stroma. It has been shown in mice that prolonged ischemia for $30 \mathrm{~min}$ is able to cause detachment of endometrial cells upon reperfusion (Okazaki et al. 2005). DíazGarcía et al. (2013) investigated the viability of transplanted uterus after exposure to warm ischemia in order to mimic the time-frame of hypoxia/ischemia that occurs during routine pelvic surgeries, and found that prolonged ischemia had severe detrimental effects on the survival of the transplanted uterus. We also found that prolonged hypoxia $(40 \mathrm{~min}) \mathrm{can}$ significantly reduce the force of myometrial contraction even after complete restoration of oxygen. This finding is consistent with the results of previous studies showing markedly reduced intestinal smooth muscle contraction after $30 \mathrm{~min}$ of sustained ischemia (Guschlbauer et al. 2010). The underlying mechanisms of the irregularity of uterine contractile activity that we observed during reoxygenation are not clear as there are limited data in the literature investigating the effect of prolonged hypoxia on contractility. However, it can be explained partly by the increased $\mathrm{Ca}^{2+}$ overload that accumulated during hypoxia. It has been demonstrated in rat cardiomyocytes that prolonged hypoxia $(60 \mathrm{~min})$ can cause significant irreversible hypercontracture and serious cellular injury during the reoxygenation, possibly due to $\mathrm{Ca}^{2+}$ accumulation in the cytosol during hypoxia (Abdallah et al. 2010). Furthermore, it was reported in isolated human uterine strips that prolonged hypoxia $(20 \mathrm{~min})$ is associated with elevated intracellular $\mathrm{Ca}^{2+}$ during entire application of hypoxia (Monir-Bishty et al. 2003). Another possible explanation for irregular myometrial activity after hypoxia is the development of fibroid tissues, inflammation, and death of some myometrial cells as we found by histologic examination. Our finding is in keeping with previous morphological examinations which showed severe intracellular damage and some cellular death during reoxygenation from sustained hypoxia in isolated rabbit (Kilgore et al. 1993) and rat (Kang et al. 2000) cardiomyocytes. When oxygen is unavailable, ATP is rapidly degraded and converted to hypoxanthine which can react with xanthine oxidase in the reoxygenation period to produce ROS (Saugstad 1996), which is known to cause muscle damage (Ballinger et al. 2000). Another detrimental effect of sustained hypoxia is the reduction of $\mathrm{Na}^{+}-\mathrm{K}^{+}$ATPase activity which was reported in hearts (Elmoselhi et al. 2003) and smooth muscles (Shen et al. 2016). The myocardium, kidney, liver, intestine, and brain are among the organs in which HPC has been studied. However, few or no studies were found relating to uterine tissue. We found that when uterine tissue was preconditioned with brief cycles of repetitive hypoxia, the degenerative endometrial and myometrial damage after the prolonged hypoxia decreased markedly in comparison with prolonged hypoxia alone. We suggest that ROS, which are produced during the prolonged hypoxia, may further damage the cellular contents. It has been shown in humans that ischemic preconditioning can significantly attenuate the detrimental endometrial dysfunction and systemic neutrophil activation in vivo (Kharbanda et al. 2001). Furthermore, it has been reported in isolated rat aortic segments that, after HPC, the level of ROS was significantly reduced and the functional recovery of endothelial cells was improved following prolonged hypoxia (Carrasco-Martín et al. 2005). The molecular mechanisms rendering slight improvement in uterine structure and function by short hypoxic insults are not fully understood. We found previously that repetitive episodes of short hypoxia can significantly improve the force of uterine contraction in pregnant rats, possibly by stimulating the prostaglandins biosynthesis and activation of the purinergic receptors (Alotaibi et al. 2015). In addition, we found that pregnant myometrium was able to withstand the effects of prolonged hypoxia than the nonpregnant tissue after repeated brief hypoxic insults (Alotaibi 2018). Other studies suggest that preconditioning with intermittent hypoxic episodes can increase ATP release and expression of its receptors which could improve muscle contraction (Elliott et al. 2013). In addition, it has been reported that preconditioning the heart with 3 cycles of ischemia can significantly prevent the reduction in $\mathrm{Na}^{+}-\mathrm{K}^{+}$ATPase activity following sustained ischemia (Elmoselhi et al. 2003).

In conclusion, this study demonstrated the detrimental effects of prolonged hypoxia on endometrial and myometrial cells survival and it clearly showed that during reoxygenation severe degenerative changes occurred in uterine tissues, which manifested as contractile dysfunction. However, challenging the uterine tissue with brief cycles of hypoxia could 
markedly improve the endometrial/myometrial structure and function after sustained hypoxia. Further studies are needed to investigate the exact mechanisms behind the HPC in the uterus as few or no studies were performed on this tissue.

Acknowledgments. I would like to thank the Deanship of Scientific Research at King Saud University for supporting this project.

Conflict of interest. None.

\section{References}

Abdallah Y, Wolf C, Meuter K, Piper H, Reusch H, Ladilov Y (2010) Preconditioning with diazoxide prevents reoxygenationinduced rigor-type hypercontracture. J. Mol. Cell. Cardiol. 48, $270-276$ https://doi.org/10.1016/j.yjmcc.2009.04.013

Alotaibi M, Arrowsmith S, Wray S (2015): Hypoxia-induced force increase (HIFI) is a novel mechanism underlying the strengthening of labor contractions, produced by hypoxic stresses. Natl. Acad. Sci. USA 112, 9763-9768 https://doi.org/10.1073/pnas.1503497112

Alotaibi MF (2017): The response of rat and human uterus to oxytocin from different gestational stages in vitro. Gen. Physiol. Biophys. 36, 75-82 https://doi.org/10.4149/gpb_2016022

Alotaibi M (2018): Brief hypoxic cycles improve uterine contractile function after prolonged hypoxia in term-pregnant but not in nonpregnant rats in vitro. Theriogenology 113, 73-77 https://doi.org/10.1016/j.theriogenology.2018.02.011

Aslan M, Senturk G E, Akkaya H, Sahin S, Yllmaz B (2017): The effect of oxytocin and Kisspeptin-10 in ovary and uterus of ischemiareperfusion injured rats. Taiwan J. Obstet. Gynecol. 56, 456-462 https://doi.org/10.1016/j.tjog.2016.12.018

Ballinger SW, Patterson C, Yan C.-N, Doan R, Burow DL, Young CG, Yakes FM, Van Houten B, Ballinger CA, Freeman BA (2000): Hydrogen peroxide-and peroxynitrite-induced mitochondrial DNA damage and dysfunction in vascular endothelial and smooth muscle cells. Circ. Res. 86, 960-966 https://doi.org/10.1161/01.RES.86.9.960

Brotanek V, Hendricks CH, Yoshida T (1969): Changes in uterine blood flow during uterine contractions. Am. J. Obstet. Gynecol. 103, 1108-1116 https://doi.org/10.1016/0002-9378(69)90515-8

Carrasco-Martín C, Alonso-Orgaz S, De la Pinta JC, Marques M, Macaya C, Barrientos A, González MM, García-Méndez A, Mateos-Cáceres PJ, Porres JC (2005): Endothelial hypoxic preconditioning in rat hypoxic isolated aortic segments. Exp. Physiol. 90, 557-569 https://doi.org/10.1113/expphysiol.2005.030163

Chanez P, Dent G, Yukawa T, Barnes P, Chung K (1990): Generation of oxygen free radicals from blood eosinophils from asthma patients after stimulation with PAF or phorbol ester. Eur. Respir. J. 3, 1002-1007

Cummins EP, Taylor CT (2005): Hypoxia-responsive transcription factors. Pflügers Arch. 450, 363-371 https://doi.org/10.1007/s00424-005-1413-7

Diaz-Garcia C, Akhi SN, Martinez-Varea A, Brannstrom M (2013): The effect of warm ischemia at uterus transplantation in a rat model. Acta Obstet. Gynecol. Scand. 92, 152-159 https://doi.org/10.1111/aogs.12027

Elliott R, Tonnu A, Ghaffar N, Taylor A, Tincello D, Norman R (2013): Enhanced purinergic contractile responses and P2X1 receptor expression in detrusor muscle during cycles of hypoxia-glucopenia and reoxygenation. Exp. Physiol. 98, 1683-1695 https://doi.org/10.1113/expphysiol.2013.075010

Elmoselhi AB, Lukas A, Ostadal P, Dhalla NS (2003): Preconditioning attenuates ischemia-reperfusion-induced remodeling of $\mathrm{Na}+-\mathrm{K}+-\mathrm{ATPase}$ in hearts. Am. J. Physiol. Heart Circ. Physiol. 285, H1055-H1063 https://doi.org/10.1152/ajpheart.00865.2002

Fageeh W, Raffa H, Jabbad H, Marzouki A (2002): Transplantation of the human uterus. Int. J. Gynaecol. Obstet. 76, 245-251 https://doi.org/10.1016/S0020-7292(01)00597-5

Gottlieb RA, Burleson KO, Kloner RA, Babior BM, Engler RL (1994): Reperfusion injury induces apoptosis in rabbit cardiomyocytes. J. Clin. Invest. 94, 1621-1628 https://doi.org/10.1172/JCI117504

Guschlbauer M, Hoppe S, Geburek F, Feige K, Huber K (2010): In vitro effects of lidocaine on the contractility of equine jejunal smooth muscle challenged by ischaemia-reperfusion injury. Equine Vet. J. 42, 53-58 https://doi.org/10.2746/042516409X475454

Gute DC, Ishida T, Yarimizu K, Korthius RJ (1998): Inflammatory responses to ischemia, and reperfusion in skeletal muscle. Mol. Cell. Biochem. 179, 169-187 https://doi.org/10.1023/A:1006832207864

Horton JW, Walker PB (1993): Oxygen radicals, lipid peroxidation, and permeability changes after intestinal ischemia and reperfusion. J. Appl. Physiol. 74, 1515-1520 https://doi.org/10.1152/jappl.1993.74.4.1515

Kang PM, Haunstetter A, Aoki H, Usheva A, Izumo S (2000): Morphological and molecular characterization of adult cardiomyocyte apoptosis during hypoxia and reoxygenation. Circ. Res. 87, 118-125 https://doi.org/10.1161/01.RES.87.2.118

Kharbanda RK, Peters M, Walton B, Kattenhorn M, Mullen M, Klein N, Vallance P, Deanfield J, MacAllister R (2001): Ischemic preconditioning prevents endothelial injury and systemic neutrophil activation during ischemia-reperfusion in humans in vivo. Circulation 103, 1624-1630 https://doi.org/10.1161/01.CIR.103.12.1624

Kilgore KS, Lucchesi BR (1993): Effect of hypoxia and reoxygenation on the isolated rabbit heart determined by monoclonal antimyosin antibody uptake. Cardiovasc. Res. 27, 1260-1267 https://doi.org/10.1093/cvr/27.7.1260

Kunt C, Kanat-Pektas M, Gungor ANC, Kurt RK, Ozat M, Gulerman C, Gungor T, Mollamahmutoglu L (2010): Randomized trial of vaginal prostaglandin E2 versus oxytocin for labor induction in term premature rupture of membranes. Taiwan J. Obstet. Gynecol. 49, 57-61 https://doi.org/10.1016/S1028-4559(10)60010-1

Kupiec-Weglinski J, Busuttil R (2005): Ischemia and reperfusion injury in liver transplantation. Transplant. Proc. 37, 1653-1656 
https://doi.org/10.1016/j.transproceed.2005.03.134

Larcombe-McDouall J, Buttell N, Harrison N, Wray S (1999): In vivo $\mathrm{pH}$ and metabolite changes during a single contraction in rat uterine smooth muscle. J. Physiol. 518, 783-790 https://doi.org/10.1111/j.1469-7793.1999.0783p.x

McCully JD, Wakiyama H, Hsieh Y.-J, Jones M, Levitsky S (2004): Differential contribution of necrosis and apoptosis in myocardial ischemia-reperfusion injury. Am. J. Physiol. Heart Circ. Physiol. 286, H1923-H1935 https://doi.org/10.1152/ajpheart.00935.2003

Monir-Bishty E, Pierce S, Kupittayanant S, Shmygol A, Wray S (2003): The effects of metabolic inhibition on intracellular calcium and contractility of human myometrium. BJOG 110, 1050-1056 https://doi.org/10.1111/j.1471-0528.2003.03103.x

Murry CE, Jennings RB, Reimer KA (1986): Preconditioning with ischemia: a delay of lethal cell injury in ischemic myocardium. Circulation 74, 1124-1136 https://doi.org/10.1161/01.CIR.74.5.1124

Okazaki M, Matsuyama T, Kohno T, Shindo H, Koji T, Morimoto Y, Ishimaru T (2005): Induction of epithelial cell apoptosis in the uterus by a mouse uterine ischemia-reperfusion model: possible involvement of tumor necrosis factor- $\alpha$. Biol. Reprod. 72, 1282-1288 https://doi.org/10.1095/biolreprod.104.035840

Sahin S, Ozakpinar OB, Ak K, Eroglu M, Acikel M, Tetik S, Uras F, Cetinel S (2014): The protective effects of tacrolimus on rat uteri exposed to ischemia-reperfusion injury: a biochemical and histopathologic evaluation. Fertil. Steril. 101, 1176-1182 https://doi.org/10.1016/j.fertnstert.2013.12.044
Saugstad OD (1996): Role of xanthine oxidase and its inhibitor in hypoxia: reoxygenation injury. Pediatrics 98, 103-107

Schafer KA, Eighmy J, Fikes JD, Halpern WG, Hukkanen RR, Long GG, Meseck EK, Patrick DJ, Thibodeau MS, Wood CE (2018): Use of severity grades to characterize histopathologic changes. Toxicol. Pathol. 46, 256-265 https://doi.org/10.1177/0192623318761348

Shen H, Liang P, Qiu S, Zhang B, Wang Y, Lv P (2016): The role of $\mathrm{Na}(+), \mathrm{K}(+)$-ATPase in the hypoxic vasoconstriction in isolated rat basilar artery. Vascul. Pharmacol. 81, 53-60 https://doi.org/10.1016/j.vph.2016.02.004

Wray S, Burdyga T, Noble D, Noble K, Borysova L, Arrowsmith S (2015): Progress in understanding electro-mechanical signalling in the myometrium. Acta Physiol. 213, 417-431 https://doi.org/10.1111/apha.12431

Wu X.-D, Zhang Z.-Y, Sun S, Li Y.-Z, Wang X.-R, Zhu X.-Q, Li W.-H, Liu X.-H (2013): Hypoxic preconditioning protects microvascular endothelial cells against hypoxia/reoxygenation injury by attenuating endoplasmic reticulum stress. Apoptosis 18, $85-98$ https://doi.org/10.1007/s10495-012-0766-6

Yukawa T, Read RC, Kroegel C, Rutman A, Chung KF, Wilson R, Cole PJ, Barnes PJ (1990): The effects of activated eosinophils and neutrophils on guinea pig airway epithelium in vitro. Am. J. Respir. Cell Mol. Biol. 2, 341-353 https://doi.org/10.1165/ajrcmb/2.4.341

Received: May 26, 2019

Final version accepted: August 2, 2019 